\title{
Etude comparative des peroxydases de dix (10) plantes supérieures couramment rencontrées au Burkina Faso
}

\author{
Mamounata DIAO $^{1 *}$, Ramatou Hawan DEMBELE ${ }^{1}$, Kiessoun KONATE $^{1,2}$ et \\ Mamoudou H. DICKO ${ }^{1}$ \\ ${ }^{1}$ Laboratoire de Biochimie, Biotechnologie, Technologie Alimentaire et Nutrition (LABIOTAN), Université \\ Joseph Ki-Zerbo, 03 BP : 848, Ouagadougou 03, Burkina Faso. \\ ${ }^{2}$ Unité de Formation et de Recherche en Sciences Appliquées et Technologiques, Université de Dédougou, BP : \\ 176, Dédougou, Burkina Faso. \\ *Auteur correspondant ; E-mail : mamounadiao@yahoo.fr; Tel :0022676531787
}

\section{RESUME}

Cette étude vise à caractériser les peroxydases de dix plantes locales à savoir Allium cepa, Brachystelma bengeri, Brassica rapa, Curculigo pilosa, Daucus carota, Phaseolus vulgaris, Sorghum bicolor, Stylochyton hypogaeus, Urginea glaucescens et Xanthosoma sagittifolium afin de trouver une alternative au raifort, l'unique source de peroxydase commerciale. Pour cela, les protéines totales ont été extraites et l'activité enzymatique a été déterminée en utilisant le gaïacol comme substrat. L'effet du pH et de la température a été étudié ainsi que les paramètres cinétiques des peroxydases. Les résultats ont montré que Brassica rapa présente la plus forte activité peroxydasique $(173,3 \mathrm{U} / \mathrm{mg})$. Les pHs optima varient entre 4,5 et 6 ; les températures optimales varient entre $30{ }^{\circ} \mathrm{C}$ et $40{ }^{\circ} \mathrm{C}$. L'étude de thermostabilité a montré que les pertes d'activité des peroxydases augmentent à la fois avec la température et la durée de chauffage. Les études cinétiques ont montré que l'affinité des peroxydases pour le gaïacol et le peroxyde d'hydrogène de même que les vitesses maximales sont plus élevées respectivement avec les peroxydases de Brassica rapa et Xanthosoma sagittifolium comparativement aux autres. Ainsi les peroxydases de Brassica rapa, Sorghum bicolor et Daucus carota se sont révélées intéressantes et comme une alternative pour d'éventuelle application.

(c) 2019 International Formulae Group. All rights reserved.

Mots clés: Peroxydase, caractérisation, plantes, gaïacol, Peroxyde d'hydrogène.

\section{Comparative study of peroxidases from ten (10) higher plants commonly found in Burkina Faso}

\begin{abstract}
This study aims to characterize the peroxidases of ten local plants namely Allium cepa, Brachystelma bengeri, Brassica rapa, Curculigo pilosa, Daucus carota, Phaseolus vulgaris, Sorghum bioclor, Stylochyton hypogaeus, Urginea glaucescens and Xanthosoma sagittifolium, in order to find an alternative to horseradish, the only source of commercial peroxidase. Thus, total proteins were extracted, and the peroxidase activity was determined using guaiacol. The effect of $\mathrm{pH}$ and temperature was studied as well as the kinetic parameters of the different peroxidases. The results show that Brassica rapa peroxidases show the highest activity (173.3
\end{abstract}


$\mathrm{U} / \mathrm{mg}$ ). Optimum pHs extracts vary between 4.5 and 6; optimal temperatures vary between $30{ }^{\circ} \mathrm{C}$ and $40{ }^{\circ} \mathrm{C}$. The study of thermostability shows that the loss of activity of all peroxidases increased with both increase of temperature and heating time. Kinetic studies have shown that the affinity per guaiacol and hydrogen peroxide as well as the maximum velocities are higher with the peroxidases of Brassica rapa and Xanthosoma sagittifolium respectively compared to other plants. Thus, peroxidases of Brassica rapa, Sorghum bicolor and Daucus carota have proved to be interesting and as an alternative for possible application.

(C) 2019 International Formulae Group. All rights reserved.

Keywords : Peroxidase, characterization, plants, guaiacol, Hydrogen peroxide.

\section{INTRODUCTION}

De nos jours, l'intérêt biotechnologique des enzymes va en croissant $\mathrm{du}$ fait des avantages économiques et environnementaux que ces dernières offrent comparativement aux catalyseurs chimiques. Parmi ces enzymes d'intérêt figurent les peroxydases [EC 1.11.1.7] qui sont des enzymes hémiques. Elles sont capables de catalyser l'oxydation d'une large gamme de composés organiques ou inorganiques, en utilisant le peroxyde d'hydrogène ou hydroperoxyde organique comme accepteur d'électron (Adeniyi et al., 2009 ; Zia et al., 2011) . Elles sont ubiquitaires chez les plantes, les animaux, les bactéries et les champignons. Chez les plantes, les peroxydases sont impliquées dans de nombreux processus physiologiques notamment dans la formation de la paroi cellulaire, la lignification et la tubérisation des cellules (Quiroga et al., 2000), dans le catabolisme de l'auxine, une hormone de croissance chez la plante. Elles participent également à la cicatrisation des tissus blessés et à la protection de la plante contre les microorganismes pathogènes (Pandey et al., 2017). Ces enzymes ont longtemps été étudiées pour leurs applications industrielles et analytiques (Agostini et al., 2002 ; Lu et al., 2016). Elles sont intensément utilisées comme réactifs dans les diagnostics cliniques et les tests ELISA (Enzyme Linked Immuno Sorbent Assay) (Agostini et al., 2002; Xu, 2016 ; Lim et al., 2018 ; Sonet et al., 2018), dans les procédés de blanchiment de la pulpe à papier et de dégradation de la lignine et aussi dans les synthèses organiques pour la production de polymères (Wang et al., 2016). Leur utilisation dans le traitement des eaux usées (Diao et al., 2011) et dans l'industrie alimentaire pour la production d'arômes naturels a été décrite (Agostini et al., 2002). Cette grande diversité d'application des peroxydases est liée d'une part à leur acceptabilité pour un grand nombre de substrats et d'autre part à leur stabilité thermique et chimique. Bien qu'elles soient ubiquitaires dans les plantes supérieures, jusqu'à nos jours, la principale source de peroxydases commerciales est la racine de raifort « horseradish » (Armoracia rusticana) (Agostini et al., 2002). Cependant, des plantes d'autres espèces peuvent fournir des peroxydases dont les caractéristiques sont identiques à celles du raifort voire meilleur. L'objectif général de cette étude est de trouver de nouvelles sources de peroxydases en vue d'applications biotechnologiques notamment dans le domaine environnemental. Spécifiquement, il s'est agi de comparer les propriétés physico-chimiques, catalytiques et cinétiques des peroxydases isolées de dix plantes supérieures couramment rencontrées au Burkina Faso (Allium cepa, Brachystelma bengeri, Brassica rapa, Curculigo pilosa, Daucus carota, Phaseolus vulgaris, Sorghum bicolor, Stylochyton hypogaeus, Urginea glaucescens, Xanthosoma sagittifolium).

\section{MATERIEL ET METHODES}

\section{Réactifs chimiques}

Le gaïacol et le Bleu de coomassie proviennent de Sigma-Aldrich. L'eau oxygénée $\left(\mathrm{H}_{2} \mathrm{O}_{2}\right)$ vient de Merck. L'acétate de sodium a été acheté à Rouses Anachemia. Le sérum albumine bovin (BSA), l'acide acétique sont de Sigma Chemical Co. Tous les autres produits chimiques sont de qualité analytique. 


\section{Matériel végétal}

Le matériel végétal étudié était constitué de dix plantes à savoir des bulbes d'Allium cepa, des gousses de Phaseolus vulgaris, des grains de Sorghum bicolor var sariasso 11, des tubercules de Brachystelma bengeri, de Brassica rapa, de Curculigo pilosa, de Daucus carota, de Stylochyton hypogaeus, de Urginea glaucescens et de Xanthosoma sagittifolium. Tous ces échantillons proviennent des marchés de Ouagadougou sauf le sorgho qui nous a été offert par le CNRST/INERA (Saria). Pour empêcher l'attaque des insectes et des moisissures, les grains de sorghum bicolor sariasso 11 ont été conservés avec la permithrine depuis leur récolte. Les neuf (09) autres échantillons ont été congelés à $-20{ }^{\circ} \mathrm{C}$ pour le stockage. Ce traitement permet une meilleure conservation des échantillons.

\section{Extraction des enzymes}

L'extraction des enzymes a été réalisée sur des tubercules, les grains germés, des gousses et des bulbes. Les grains de sorgho (Sorghum bicolor) ont été d'abord lavés avec l'eau distillée, puis mis à germination pendant 72 heures à la température ambiante (25-30 $\left.{ }^{\circ} \mathrm{C}\right)$ en les arrosant et les remuant régulièrement. La germination a été faite à l'obscurité pour minimiser la photosynthèse. Les grains germés ont été rendus en poudre fine et les neuf autres échantillons ont été broyés séparément, afin d'obtenir des pâtes homogènes. Les farines de sorgho et les pâtes des neuf autres échantillons ont servi enfin pour l'extraction des enzymes. Les extraits enzymatiques ont été obtenus en mélangeant $250 \mathrm{mg}$ d'organes (préalablement broyés) avec $1,5 \mathrm{ml}$ de tampon Tris-HCI $50 \mathrm{mM}, \mathrm{pH}$ 7,3 contenant $0,5 \mathrm{M}$ de chlorure de calcium et $5 \mathrm{mM}$ du $\beta$-mercapto-éthanol à $4{ }^{\circ} \mathrm{C}$. Le mélange a été agité et laissé au repos à $4{ }^{\circ} \mathrm{C}$ pendant une heure. L'homogénat a ensuite été centrifugé (14000 g, $\left.4{ }^{\circ} \mathrm{C}, 45 \mathrm{~min}\right)$ et le surnageant recueilli. Une seconde extraction a été faite sur le précipité en y ajoutant $1 \mathrm{ml}$ du tampon d'extraction. Les deux surnageants ont été mélangés pour constituer l'extrait enzymatique.

\section{Dosage des protéines solubles Préparation de la solution de CBBG-250}

La solution de CBBG-250 (Sigma nr. B-1131) ou réactif de Sedmak a été préparée à une concentration de $0,06 \%(\mathrm{p} / \mathrm{v})$ dans une solution d'acide perchlorique à $3 \%(\mathrm{p} / \mathrm{v})$, laissée sous agitation pendant $24 \mathrm{~h}$ puis filtrée. La densité optique du mélange a été ajustée avec une solution d'acide perchlorique 3\% $(\mathrm{p} / \mathrm{v})$ au cas où elle ne serait pas comprise entre 1,3 et 1,5 à $450 \mathrm{~nm}$. Le réactif ainsi préparé a été stocké à l'abri de la lumière jusqu'à utilisation.

\section{Dosage des protéines}

Les protéines des extraits ont été quantifiées par la méthode de linéarisation de Bradford utilisant le ratio A620/A450 en fonction de la concentration en protéines. Les manipulations ont été adaptées à un lecteur de plaque ELISA et le dosage a été effectué comme suit : à $10 \mu 1$ de l'extrait, on ajoute 90 $\mu \mathrm{l}$ d'eau distillée et $100 \mu \mathrm{l}$ de la solution de CBBG-250. Le volume final de chaque puits est de $200 \mu \mathrm{l}$ et les lectures de DO ont été effectuées en triplicata. Les lectures ont été faites à $450 \mathrm{~nm}$ et $620 \mathrm{~nm}$ pour chaque échantillon et le rapport A620/A450 a été utilisé pour la quantification des protéines. Un contrôle a été effectué pour chaque échantillon en remplaçant l'extrait enzymatique par l'eau distillée. Le sérum albumine bovine $(100 \mu \mathrm{g} / \mathrm{ml})$ a servi de standard.

\section{Détermination de l'activité des peroxydases (POX)}

L'activité des peroxydases a été mesurée à $25 \quad{ }^{\circ} \mathrm{C}$ au spectrophotomètre (SPECTRONIC R GENESYS TM 5) en suivant l'oxydation de l'acide 3-méthoxyphénol (gaïacol) en présence de $\mathrm{H}_{2} \mathrm{O}_{2}$. Le milieu réactionnel était composé de $10 \mu \mathrm{l}$ de l'extrait enzymatique (dilué ou non dans une solution d'extraction) ajoutés à $170 \mu \mathrm{ldu}$ tampon citrate-phosphate $100 \mathrm{mM}$, pH 5 et 10 $\mu \mathrm{l}$ de gaïacol $100 \mathrm{mM}$. La réaction enzymatique démarre dès qu'on ajoute $10 \mu \mathrm{l}$ $\mathrm{d}^{\prime} \mathrm{H}_{2} \mathrm{O}_{2} 100 \mathrm{mM}$. Les mesures ont été effectuées en triplicata et le volume final des puits était de $200 \mu$ l. Un contrôle a été 
effectué pour chaque échantillon en remplaçant l'extrait enzymatique ou le substrat par le tampon. La réaction a été suivie à $470 \mathrm{~nm}$. Une unité d'activité enzymatique (U) est définie comme étant la quantité d'enzyme libérant un $\mu \mathrm{mol}$ de 3,3'dimethoxy-4,4'-biphenoquinone par minute.

Les résultats ont été exprimés en activités spécifiques $(\mathrm{U} / \mathrm{mg})$ selon l'équation suivante :

$\mathrm{A}(\mathrm{U} / \mathrm{mg})=\frac{\Delta \mathrm{DO} \times \varepsilon^{-1} \times \mathrm{L}^{-1} \times \mathrm{VT}}{\text { Quantité de protéines }(\mathrm{mg})} \times$ Facteur de dilution

$\Delta \mathrm{DO}=$ pente de la portion linéaire de la courbe de suivi de la cinétique exprimée en $\mathrm{DO} / \mathrm{min}$

$\varepsilon=$ coefficient d'extinction molaire $\left(\varepsilon 470=5200 \mathrm{~L} \mathrm{~mol}^{-1} \mathrm{~cm}^{-1}\right) ; \mathrm{L}=$ longueur $\mathrm{du}$ trajet optique

$\mathrm{V}_{\mathrm{T}}=$ Volume total.

\section{Détermination de l'activité en fonction du pH}

L'influence du $\mathrm{pH}$ sur l'activité des peroxydases a été étudiée en mesurant les activités à $470 \mathrm{~nm}$ au spectrophotomètre après 5 min d'incubation de l'enzyme avec ses substrats gaïacol et $\mathrm{H}_{2} \mathrm{O}_{2}$ dans du tampon citrate-phosphate $100 \mathrm{mM}, \mathrm{pH} 3$ à 7. Les essais ont été effectués en duplicata à $25^{\circ} \mathrm{C}$, à chaque $\mathrm{pH}$.

\section{Détermination de l'activité en fonction de la température \\ Détermination des températures optimales}

La température optimale d'activité des peroxydases a été étudiée dans les conditions standards à $470 \mathrm{~nm}$ dans du tampon citratephosphate $100 \mathrm{mM}, \mathrm{pH} 5$, à des températures variant de 0 à $90{ }^{\circ} \mathrm{C}$ avec $10^{\circ} \mathrm{C}$ d'intervalle.

\section{Détermination de la stabilité thermique}

Des aliquotes de $100 \mu \mathrm{l}$ de chaque extrait enzymatique ont été incubées pendant $10 \mathrm{~min}$ et $30 \mathrm{~min}$ à différentes températures $\left(50{ }^{\circ} \mathrm{C}\right.$ à $\left.90{ }^{\circ} \mathrm{C}\right)$. Après incubation, $10 \mu \mathrm{l}$ d'extrait ont été prélevés pour déterminer l'activité résiduelle à $\mathrm{pH} 5$. La variation des températures était de $10{ }^{\circ} \mathrm{C}$.
Détermination de l'efficacité catalytique et des vitesses maximales apparentes

La constante de Michaelis (Km) et la vitesse maximale $(\mathrm{Vm})$ ont été déterminées pour le gaïacol puis pour le peroxyde d'hydrogène à des concentrations allant de 0,01-0,1 M. Pour déterminer la Km et la Vm pour le gaïacol, la concentration du peroxyde d'hydrogène a été maintenue constante à 0,1 $M$ tandis que celle du gaïacol a été variée de 0,01-0,1 M. Pour la détermination de $\mathrm{Km}$ et Vm pour le peroxyde d'hydrogène, le même protocole a été suivi. Les constantes cinétiques ont été déterminées avec le tracé en double inverse de Lineweaver-burk.

\section{Analyse statistique}

L'analyse statistique a été réalisée à l'aide de SPSS version 20. Toutes les analyses ont été effectuées en triplicata et les résultats ont été exprimés en moyenne \pm écart type. Les données ont été soumis au Test de Levene pour vérifier l'égalité des variances suivie du test $t$ de student pour rechercher une différence significative $(\mathrm{p}<0,05)$ entre les peroxydases des différents extraits de plantes.

\section{RESULTATS}

\section{L'activité enzymatique des peroxydases}

Les résultats des activités peroxydasiques des extraits de 10 plantes ainsi que leurs concentrations protéiques sont consignés dans le Tableau 1. Les activités spécifiques varient significativement d'une plante à l'autre. La plus forte activité est observée chez Brassica rapa (173,3 $\pm 14,16$ $\mathrm{U} / \mathrm{mg}$ ) et la plus faible activité chez Curculigo pilosa $(0.9 \pm 0.29 \mathrm{U} / \mathrm{mg})$. Outre Brassica rapa, les plantes présentant des activités remarquables sont Daucus carota, Sorghum bicolor var. $11, U$. glaucescens et Xanthosoma sagittifolium où les activités sont $109,8 \pm 9,35 ; \quad 80,9 \pm 5,47 ; \quad 36,6 \pm 1,05$ et $31,2 \pm 1,55 \mathrm{U} / \mathrm{mg}$ respectivement. L'activité de Brassica rapa est 10 fois supérieure à celles de $B$. bengeri et plus de vingt 20 fois supérieure à celles de $S$. hypogaeus, C. pilosa et A. cepa. 


\section{pH optimum des peroxydases des différentes plantes}

L'activité des peroxydases des dix plantes a été mesurée à différents $\mathrm{pH}$ en utilisant le gaïacol comme substrat. Il ressort de cette étude que les pHs optima d'activité des peroxydases de tous les extraits sont acides et varient entre 4,5 et 6 (Tableau 2). Le $\mathrm{pH}$ le plus faible $(4,5)$, est observé chez $B$. bengeri tandis que le $\mathrm{pH}$ le plus élevé (6), est observé chez $P$. vulgaris. Contrairement aux peroxydases de A. cepa $(5-6,5)$ et $B$. rapa (46) qui sont stables sur une large gamme de $\mathrm{pH}$, les peroxydases de $U$. glaucescens sont très sensibles aux variations de $\mathrm{pH}$. Une inhibition de plus de $90 \%$ est observée aux pHs inférieurs à 4,5 et aux $\mathrm{pH}$ supérieurs à 5,5 sur les peroxydases de cette dernière. On note également des activités optimales au $\mathrm{pH} 5$ avec les peroxydases de cinq plantes à savoir $U$. glaucescens, B. bengeri, S. hypogaeus, $X$. sagittifolium et D. carota.

\section{Températures optimales des différentes peroxydases}

Les températures optimales des peroxydases des plantes sont résumées dans le Tableau 2. Parmi les peroxydases des dix plantes sélectionnées, cinq ont une température optimale autour de $30{ }^{\circ} \mathrm{C}$ et les cinq autres, une température optimale autour de $40{ }^{\circ} \mathrm{C}$. On note que les extraits peroxydasiques de A. cepa, S. hypogaeus, D. carota, $S$. bicolor et $P$. vulgarus ont une température optimale inférieure à celle de $U$. glaucescens, C. pilosa, X. sagittifolium, B. rapa et $B$. bengeri.

\section{Etude de la thermostabilité des extraits peroxydasiques}

Les peroxydases des dix plantes soumises à des températures variant de 50 à $90{ }^{\circ} \mathrm{C}$ pendant $10 \mathrm{~min}$ et $30 \mathrm{~min}$ ont présenté des stabilités différentes (Figures 1 et 2). Les pertes d'activité des peroxydases augmentaient à la fois avec la température et la durée de chauffage. Après $10 \mathrm{~min}$ d'incubation à $50{ }^{\circ} \mathrm{C}$, seules les peroxydases Urginea glaucescens conservent toutes leur activité. Les peroxydases des 9 autres perdent entre 1 et $65 \%$ de leur activité enzymatique (Figure 1A). La plus faible activité peroxydasique est observée chez Sorghum bicolor qui ne conserve que $35 \%$ de son activité. Cinq plantes conservent plus de 50\% de leurs activités; par ordre décroissant de thermostabilité à savoir Urginea glaucescens, Brassica rapa, Phaseolus vulgaris, Brachystelma bengri et Stylochyton hypogaeus. Après 30 min d'incubation à 50 ${ }^{\circ} \mathrm{C}$, il y a une baisse importante d'activité chez C. pilosa, D. carota, S. bicolor et X. sagittifolium (Figure 2A). Seuls Stylochyton hypogaeus, Brachystelma bengeri et Phaseolus vulgaris conservent plus de 50\% de leur activité enzymatique. Chez d'autres plantes, il y a une augmentation de l'activité par rapport à celle obtenue après $10 \mathrm{~min}$, il s'agit de Allium cepa dont l'activité passe de $35 \%$ à $47 \%$, de Stylochyton hypogaeus dont l'activité passe de $65 \%$ à $81 \%$ et de Brachystelma bengeri dont l'activité enzymatique passe de $76 \%$ à $93 \%$; chez Urginea glaucesces, il y a une conservation de l'activité. Après incubation à $60{ }^{\circ} \mathrm{C}$ pendant $10 \mathrm{~min}$, seuls Brassica rapa, Brachystelma bengeri et Urginea glaucescens conservent plus de 50\% de leurs activités (Figure 1A). Après 30 min d'incubation à $60^{\circ} \mathrm{C}$, seules les peroxydases de Brachystelma bengeri conservent plus de $50 \%$ de leur activité (53\%) (Figure 1B). Après 10 min à $70{ }^{\circ} \mathrm{C}$ toutes les peroxydases perdent plus de la moitié de leurs activités (Figure 1B). Au-delà de $80^{\circ} \mathrm{C}$, les peroxydases des dix plantes perdent presque toutes leurs activités (Figures 1C et 2C). Les peroxydases de $D$. carota se révèlent être les plus thermostables à $80{ }^{\circ} \mathrm{C}$ et $90{ }^{\circ} \mathrm{C}$ en conservant au moins $10 \%$ de leurs activités après 30 min (Figure 2C). Par contre elle est la plus affecté à $50{ }^{\circ} \mathrm{C}$. La peroxydase de Urginea glaucescens, bien qu'elle soit plus active à $50{ }^{\circ} \mathrm{C}$ se montre très sensible aux variations de température.

\section{Les constantes de Michaelis $(\mathrm{Km})$ et les vitesses maximales $(\mathrm{Vm})$}

Les constantes cinétiques ont été déterminées avec différentes concentration en gaïacol et en peroxyde d'hydrogène. Les 
résultats obtenus à partir du tracé en double inverse de Lineweaver-Burk sont reportés dans le Tableau 3. La plus grande $\mathrm{Km}$ pour le gaïacol est obtenu avec les peroxydases de Brachystelma bengeri $(80,12 \pm 7,93 \mathrm{mM})$. Les plus petites $\mathrm{Km}$ sont obtenues avec les peroxydases de Daucus carota $(0,38 \pm 0,16$ $\mathrm{mM})$ et $P$. vulgaris $(0,49 \pm 0,17 \mathrm{mM})$. Les sept autres ont présenté des $\mathrm{Km}$ comprise entre $0,977 \pm 0,16 \mathrm{mM}$ et $4,16 \pm 0,16 \mathrm{mM}$. Quant aux $\mathrm{Km}$ de réduction du peroxyde d'hydrogène, les valeurs sont beaucoup plus faibles comparativement à celle du gaïacol et varient de $0,003 \pm 0,002$ à $0,86 \pm 0,17 \mathrm{mM}$. Les plus grandes valeurs sont obtenues avec les peroxydases de $B$. rapa et les plus faible avec B. bengeri.
Pour ce qui est des Vm d'oxydation du gaïacol, Brassica rapa a la valeur la plus élevée $(312,74 \pm 2,91 \mu \mathrm{M} / \mathrm{min})$, soit environ 4 fois celle de Daucus carota $(89,64 \pm 1,52$ $\mu \mathrm{M} / \mathrm{min}$ ) qui est la deuxième plante présentant une $\mathrm{Vm}$ élevée et celle de $B$. bengeri $(76,92 \pm 0,58 \mu \mathrm{M} / \mathrm{min})$, la troisième. La plus faible valeur $(0,15 \pm 0,02 \mu \mathrm{M} / \mathrm{min})$ est obtenue avec $C$. pilosa. Pour les $\mathrm{Vm}$ de réduction du peroxyde d'hydrogène, Il ressort que les peroxydases de Xanthosoma sagittifolium réduisent le peroxyde d'hydrogène avec une Vm nettement supérieure à celles des autres peroxydases et les peroxydases de Curculigo pilosa ont la plus faible $\mathrm{Vm}$.

Tableau 1: Activité des peroxydases et concentrations protéiques dans les plantes.

\begin{tabular}{llllll}
\hline Échantillon & $\begin{array}{l}\text { Volume } \\
(\mathbf{m l})\end{array}$ & $\begin{array}{l}\text { Protéines } \\
(\mathbf{m g})\end{array}$ & $\begin{array}{l}\text { Activité } \\
(\mathbf{U I})\end{array}$ & $\begin{array}{l}\text { Activité spécifique } \\
(\mathbf{U I} / \mathbf{m g})\end{array}$ & $\begin{array}{l}\text { Activité par g du } \\
\text { végétal }(\boldsymbol{\mu m o l} / \mathbf{g})\end{array}$ \\
\hline C. pilosa & 2 & 37,9 & 35,2 & $0,9 \pm 0,29^{\mathrm{f}}$ & 140,9 \\
\hline S. hypogaeus & 1,9 & 588,0 & 563,3 & $1,0 \pm 0,61^{\mathrm{f}}$ & 2253,1 \\
\hline A. cepa & 2,1 & 29,7 & 49,8 & $1,7 \pm 0,34^{\mathrm{f}}$ & 199,4 \\
\hline B. bengeri & 1,8 & 19,5 & $93,6 \mathrm{~g}$ & $4,8 \pm 0,51^{\mathrm{f}}$ & 374,3 \\
\hline U. glaucescens & 2 & 19,8 & 724,68 & $36,6 \pm 1,05^{\mathrm{d}}$ & 2898,72 \\
\hline P. vulgaris & 2 & 133,7 & 2321,4 & $17,4 \pm 2,11^{\mathrm{e}}$ & 9285,6 \\
\hline D. carota & 2 & 88,2 & 9686,7 & $109,8 \pm 9,35^{\mathrm{b}}$ & 38746,8 \\
\hline X. sagittifolium & 1,8 & 55,1 & 1722,7 & $31,2 \pm 1,55^{\mathrm{d}}$ & 6890,7 \\
\hline S. bicolor var. 11 & 1,8 & 81,2 & 6573,6 & $80,9 \pm 5,47^{\mathrm{c}}$ & 26294,3 \\
\hline B. rapa & 2 & 64,8 & 11229,2 & $173,3 \pm 14,16^{\mathrm{a}}$ & 44916,8 \\
\hline Les & &
\end{tabular}

Les moyennes des activités spécifiques non suivies par une même lettre minuscule sont statistiquement différentes à un seuil de $5 \%(P<0,05)$.

Tableau 2: $\mathrm{pH}$ optimum et température optimale des peroxydases des plantes.

\begin{tabular}{lll}
\hline Echantillons & PH & Température \\
\hline U. glaucescens & 5 & 40 \\
\hline B. bengeri & $4,5-5$ & 40 \\
\hline A. cepa & $5-5,5$ & 30 \\
\hline C. pilosa & 5,5 & 40 \\
\hline S. hypogaeus & $5-5,5$ & 30 \\
\hline S. bicolor & $5,5-6$ & 30 \\
\hline Phaseolus vulgaris & 6 & 30 \\
\hline X. sagittifolium & 5 & 40 \\
\hline Daucus carota & 5 & 30 \\
\hline Brassica rapa & $5,5-6$ & 40 \\
\hline
\end{tabular}



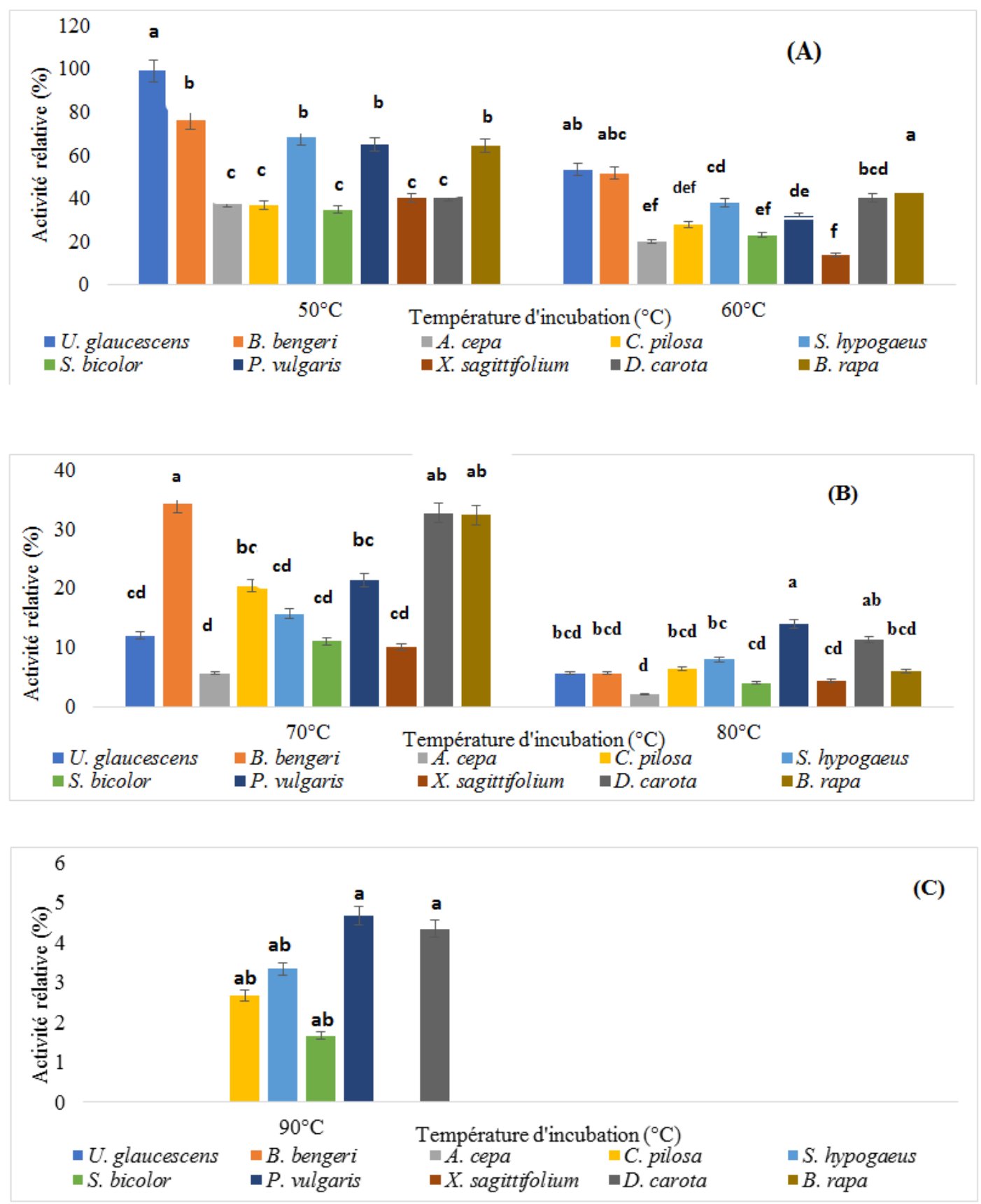

Figure 1 : Etudes de la thermostabilité des peroxydases des différentes plantes après 10 min de préincubation aux températures variant de $50{ }^{\circ} \mathrm{C}$ à $90^{\circ} \mathrm{C}$.
(A) Activité relative à $50^{\circ} \mathrm{C}$ et $60^{\circ} \mathrm{C}$
(B) Activité relative à $70^{\circ} \mathrm{C}$ et $80^{\circ} \mathrm{C}$
(C) Activité relative à $90^{\circ} \mathrm{C}$ 

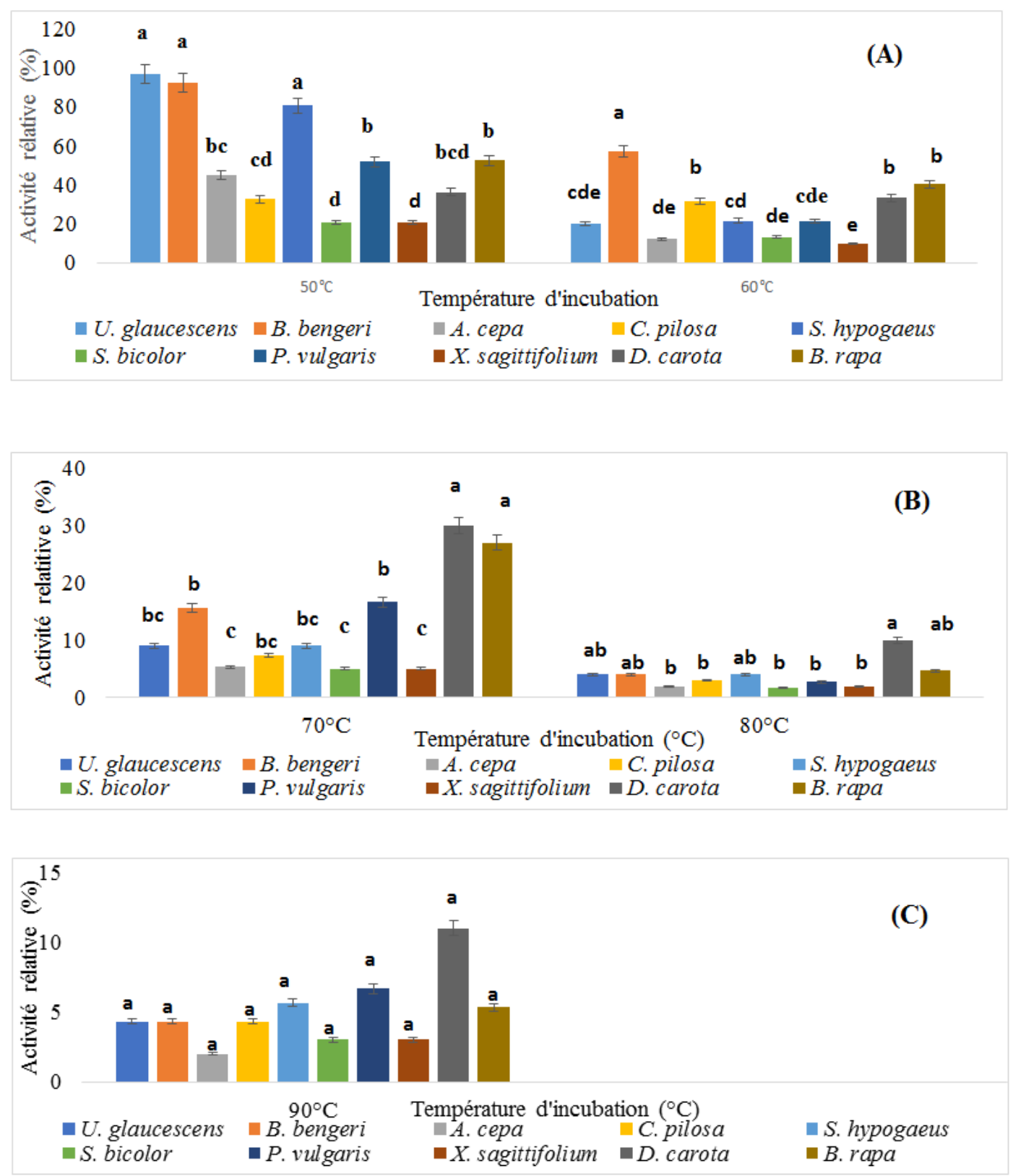

Figure 2 : Etudes de la thermostabilité des peroxydases des différentes plantes après 30 min de préincubation aux températures variant de $50{ }^{\circ} \mathrm{C}$ à $90^{\circ} \mathrm{C}$.
(A) Activité relative à $50^{\circ} \mathrm{C}$ et $60^{\circ} \mathrm{C}$
(B) Activité relative à $70^{\circ} \mathrm{C}$ et $80^{\circ} \mathrm{C}$
(C) Activité relative à $90^{\circ} \mathrm{C}$ 
Tableau 3: paramètre cinétique des peroxydases des différentes plantes.

\begin{tabular}{lllll}
\hline Echantillons & $\begin{array}{l}\text { Vm du gaïacol } \\
(\boldsymbol{\mu M} / \mathbf{m i n})\end{array}$ & $\begin{array}{l}\text { Km du gaïacol } \\
(\mathbf{m M})\end{array}$ & $\begin{array}{l}\mathbf{V m ~ d e ~} \mathbf{H}_{2} \mathbf{O}_{2} \\
(\boldsymbol{\mu M} / \mathbf{m i n})\end{array}$ & $\begin{array}{l}\mathbf{K m ~ d e ~} \mathbf{H}_{2} \mathbf{O}_{2} \\
(\mathbf{m M})\end{array}$ \\
\hline U. glaucescens & $6,43 \pm 0,17 \mathrm{a}$ & $2,43 \pm 0,45 \mathrm{a}$ & $2,43 \pm 0,3 \mathrm{a}$ & $0,15 \pm 0,04 \mathrm{a}$ \\
\hline B. bengeri & $76,92 \pm 0,58 \mathrm{~b}$ & $80,12 \pm 7,93 \mathrm{~b}$ & $1,23 \pm 0,05 \mathrm{~b}$ & $0,003 \pm 0,002 \mathrm{~b}$ \\
\hline A. cepa & $0,31 \pm 0,02 \mathrm{c}$ & $1,03 \pm 0,12 \mathrm{c}$ & $0,33 \pm 0,04 \mathrm{c}$ & $0,55 \pm 0,14 \mathrm{c}$ \\
\hline C. pilosa & $0,15 \pm 0,02 \mathrm{~d}$ & $1,25 \pm 0,14 \mathrm{c}$ & $0,21 \pm 0,02 \mathrm{c}$ & $0,12 \pm 0,05 \mathrm{a}$ \\
\hline S. hypogaeus & $1,5 \pm 0,15 \mathrm{e}$ & $4,16 \pm 0,16 \mathrm{e}$ & $0,26 \pm 0,026 \mathrm{c}$ & $0,006 \pm 0,004 \mathrm{~b}$ \\
\hline S. bicolor & $8,72 \pm 0,3 \mathrm{a}$ & $1,51 \pm 0,5 \mathrm{~d}$ & $0,13 \pm 0,06 \mathrm{~d}$ & $0,005 \pm 0,0017 \mathrm{~b}$ \\
\hline P. vulgaris & $26,43 \pm 0,5 \mathrm{~g}$ & $0,49 \pm 0,17 \mathrm{f}$ & $1,36 \pm 0,02 \mathrm{a}$ & $0,004 \pm 0,001 \mathrm{~b}$ \\
\hline$X$. sagittifolium & $17,45 \pm 1,67 \mathrm{~h}$ & $1,83 \pm 0,29 \mathrm{ad}$ & $8,26 \pm 0,013 \mathrm{e}$ & $0,005 \pm 0,002 \mathrm{~b}$ \\
\hline D. carota & $89,64 \pm 1,52 \mathrm{j}$ & $0,38 \pm 0,16 \mathrm{f}$ & $7,75 \pm 0,18 \mathrm{f}$ & $0,01 \pm 0,003 \mathrm{~d}$ \\
\hline B. rapa & $312,74 \pm 2,91 \mathrm{k}$ & $0,977 \pm 0,16 \mathrm{~d}$ & $18,65 \pm 0,046 \mathrm{j}$ & $0,86 \pm 0,17 \mathrm{c}$
\end{tabular}

Dans chaque colonne les moyennes non suivies par une même lettre minuscule sont statistiquement différentes à un seuil de $5 \%(P<0,05)$.

\section{DISCUSSION}

Dans cette étude, des peroxydases de dix (10) plantes ont été comparées sur la base de leurs propriétés catalytiques et cinétiques. Parmi ces plantes, Brassica rapa a présenté l'activité peroxydasique $(173,3 \mathrm{U} / \mathrm{mg})$ la plus élevée. La comparaison de l'activité peroxydasique de cette dernière a celle d'autres plantes montre qu'elle a une activité intéressante. Ainsi des activités inferieures ont été reportées chez d'autres plantes à savoir, le blé (Triticum aestivum ssp vulgare) dont l'activité est de 0,02 U/mg (Altin et al., 2017), le palmier (Phoenix dactylifera L.) dont l'activité spécifique est de 53,3 U/mg (AlSenaidy et Ismael, 2011). Cependant, des activités supérieures ont été rapportées chez d'autres plantes à savoir radis (Raphanus sativus) dont l'activité spécifique est de 226 U/mg (Diao et al., 2018), Metroxylon sagu et Spondias tuberosa dont les activités spécifiques sont supérieures à $800 \mathrm{U} / \mathrm{mg}$ (Onsa et al., 2004; Pinto et al., 2015). L'activité spécifique des peroxydases de Daucus carota est de 109,8 U/mg. Ce résultat est largement supérieur à celui $(2,1 \mathrm{U} / \mathrm{mg})$ rapporté par Santos de Araujo et al. (2004) sur la même plante. Ces variations pourraient être attribuées à l'influence des pratiques culturales et des conditions de mise en culture, responsables de l'état de stress oxydatif chez les plantes et par conséquent augmentent ou inhibent des enzymes oxydatives (Mbouobda et al., 2014 ; Diop et al., 2019). L'activité spécifique des peroxydases des grains germés de sorghum bicolor var.sariasso 11 est de 81 U.mg ${ }^{-1}$. Cette valeur est inférieure à celle $(68,6 \mathrm{U} / \mathrm{mg})$ de la variété sariasso 10 obtenue dans nos travaux antérieurs (Diao et al., 2011). Une étude réalisée au Burkina Faso a démontré que l'activité des peroxydases des grains de sorgho dépend de la variété utilisée. Parmi les variétés couramment rencontrées, Sariasso 11 a une activité peroxydasique non négligeable (Dicko et al., 2002). Des faibles activités spécifiques sont observées chez Allium cepa $(1,68 \mathrm{U} / \mathrm{mg})$. Nos résultats corroborent ceux de Benkeblia et Shiomi (2004) dans le cas de la même espèce. Des activités identiques à celles de Urginea glaucescens ont été obtenues par Santos de Araujo et collaborateurs en 2004 sur Solanum avicular. Des valeurs proches de l'activité des peroxydases de Curculigo pilosa et Stylochyton hypogaeus ont été observées dans la racine de Daucus carota (Santos de Araujo et al., 2004). Quant à B. bengeri, son activité peroxydasique est proche de celle de la glutathion-peroxydase obtenu par Nwobodo et al. (2018). 
Les pH optima d'activité des peroxydases de tous nos extraits sont acides et varient entre 4,5 et 6 . Ces résultats sont différents de celui de la peroxydase cationique $\mathrm{C}_{S}$ de Raphanus sativus, obtenue par Kim et Lee (2005) avec le o-dianisidine, comme substrat. Cette différence serait due au substrat donneur d'électron utilisé par ces derniers. En effet, avec le gaïacol comme substrat, des $\mathrm{pH}$ optima acides ont été rapportés pour les peroxydases présentes dans les tissus de nombreuses plantes (Mika et luïthje, 2003 ; Tao et al., 2018). Les pertes d'activité constatées aux faibles $\mathrm{pH}$ pourraient être dues au détachement de l'hème de la chaîne polypeptidique, également au $\mathrm{pH}$ élevé, elles pourraient résulter d'une dénaturation des protéines enzymatiques (Kim et Lee, 2005). Des pH optima (6) similaires à ceux de Phaseolus vulgaris et chez Brassica rapa ont été observés chez la peroxydase du cresson (Lepidium sativum sub sp. Sativum) par Altay et al., 2018. Il en est de même pour les peroxydases de B. bengeri, $S$. hypogaeus, $U$. glaucescens, $D$ carota et $X$. sagittifolium qui ont des $\mathrm{pH}$ identiques (5) à celui de l'isoenzyme anionique de Raphanus sativus obtenu par Wang et al. (2004). Les peroxydases de Brachystelma bengeri, Allium cepa, Stylochyton hypogaeus, Sorghum bicolor et Curculigo pilosa ont des $\mathrm{pH}$ optima un peu plus larges que les autres. Ceci leur confère un avantage particulier.

Toutes les plantes étudiées ont une température optimale autour de $30-40{ }^{\circ} \mathrm{C}$. Les peroxydases apoplastiques, cytosoliques et solubles de plusieurs plantes exhibent des températures optimales entre $30{ }^{\circ} \mathrm{C}$ et $60{ }^{\circ} \mathrm{C}$ (Neves, 2002 ; Altin et al., 2017). Après 10 min d'incubation à $50{ }^{\circ} \mathrm{C}$, seule la peroxydase de Urginea glaucescens conserve toute son activité. Les peroxydases des neuf autres perdent entre 1 et $65 \%$ de leurs activités enzymatiques. Cette rapide perte d'activité pourrait être due à l'inactivation d'isoformes labiles. En effet, les isoformes labiles et résistants peuvent coexister dans les plantes (Anthon et Barette, 2002), l'activité résiduelle serait due à ces isoformes résistantes. La plus faible activité peroxydasique est observée chez Sorghum bicolor qui ne conserve que $35 \%$ de son activité tandis que la plus forte activité est observée chez Urginea glaucescens qui conserve la totalité de son activité. Des résultats semblables ont été obtenus sur des peroxydases isolées de la racine du maïs qui perdent entre 40 et $50 \%$ de leurs activités après $5 \mathrm{~min}$ à $50{ }^{\circ} \mathrm{C}$ (Mika et Lüthje, 2003). Après 30 min à $50{ }^{\circ} \mathrm{C}$, seuls Stylochyton hypogaeus, Phaseolus vulgaris et Brachystelma bengeri conservent plus de 50\% de leurs activités enzymatiques, cela permet de déduire que les peroxydases de ces plantes ont une thermostabilité élevée. Après incubation à $60{ }^{\circ} \mathrm{C}$ pendant $10 \mathrm{~min}$, seuls Brachystelma bengeri et Urginea glaucescens conservent plus de 50\% de leurs activités. Des résultats similaires ont été obtenus avec l'isoenzyme A2 de la peroxydase de Brassica napus qui conserve $50 \%$ de son activité après 10 min à $65^{\circ} \mathrm{C}$ (Agostini et al., 2002). Après 30 min d'incubation à $60 \quad{ }^{\circ} \mathrm{C}$, seul Brachystelma bengeri conserve plus de 50\% de son activité. La peroxydase de cette dernière est plus stable que celle de Daucus carota obtenu par Pinto et al. (2015), qui perd $70{ }^{\circ} \mathrm{C}$ de son activité après 10 min à $60^{\circ} \mathrm{C}$. $\mathrm{Au}$ vu de tous ces résultats, nous pouvons conclure que la peroxydase de Brachystelma bengeri est la plus thermostable suivie de Urginea glaucescens tandis que celle de $C$. pilosa et $S$. bicolor sont les moins thermostables.

Une KM faible dans la réaction reflète la plus grande affinité de l'enzyme pour son substrat, tandis qu'une KM plus élevé reflète la plus faible affinité pour son substrat. Les $\mathrm{Km}$ pour le peroxyde d'hydrogène sont inférieurs à celles obtenues avec le gaïacol, indiquant que les peroxydases extraites des différentes plantes ont plus d'affinité pour le peroxyde d'hydrogène que pour le gaïacol qui est le donneur d'électrons. Des résultats similaires ont été obtenus pour la peroxydase de Metroxylon sagu qui a montré une plus forte affinité pour le peroxyde d'hydrogène que pour le gaïacol (Onsa et al., 2004). Cependant d'autres résultats différents montrant une plus forte affinité pour le donneur d'électron (o-dianisidine) que pour 
l'accepteur d'électron (peroxyde d'hydrogène) ont été reportés par Neves en 2002. Quand bien même le gaïacol est couramment utilisé comme substrat des peroxydases, il est important de souligner que pour les substrats phénoliques, l'acide férulique (Leon et al., 2002), la o-dianisidine (Neves en 2002 ; AlSenaidy et Ismael 2011) sont aussi de bons substrats pour les peroxydases. Les Vm d'oxydation du gaïacol et de réduction du peroxyde d'hydrogène varient d'une plante à l'autre. Les plus grandes valeurs ont été obtenues avec $B$. rapa. Elles sont de $18,65 \pm 0,046 \mathrm{U} / \mathrm{ml}$ et de $312,74 \pm 2,91 \mathrm{U} / \mathrm{ml}$ respectivement pour le peroxyde d'hydrogène et le gaïacol. Ces valeurs sont largement inférieures à celles obtenues $(727,11 \mathrm{U} / \mathrm{ml}$ et $1217,1 \mathrm{U} / \mathrm{ml}$ ) par Altay et al. (2018) respectivement pour ces substrats avec les peroxydases de Lepidium sativum sub sp. sativum.

\section{Conclusion}

Dans cette étude, dix plantes ont été comparées sur la base de leurs propriétés catalytiques et cinétiques. Il ressort que Brassica rapa présente la plus grande activité peroxydasique, suivi par Daucus carota. Les plantes comme Sorghum bicolor, Urginea glaucescens et Xanthosoma sagittifolium présentent également des activités non négligeables. Toutes ces peroxydases ont une activité relativement plus élevée en milieu faiblement acide, entre $\mathrm{pH} 4,5$ et 6 . Leurs températures optimales varient entre $30{ }^{\circ} \mathrm{C}$ et $40{ }^{\circ} \mathrm{C}$ selon la plante. L'étude de la thermostabilité montre que les peroxydases de Brachystelma bengeri sont les plus thermostables. Les peroxydases de Brassica rapa et de Urginea glaucescens exhibent aussi une thermostabilité assez intéressante. L'affinité des peroxydases pour le gaïacol demeure faible pour tous les extraits. Contrairement au gaïacol, le peroxyde d'hydrogène est un bon substrat pour toutes ces enzymes. Ces informations obtenues pourraient servir pour la purification et les applications biotechnologiques de ces enzymes.

\section{CONFLIT D'INTERETS}

Les auteurs déclarent qu'il n'existe aucun conflit d'intérêt pour cet article.

\section{CONTRIBUTIONS DES AUTEURS}

$M D$ et RHD ont participé à la production des résultats. $\mathrm{KK}$ a intervenu dans la relecture et la correction du manuscrit et MHD a encadré scientifiquement les travaux depuis l'élaboration du thème jusqu'à la rédaction du document final.

\section{REMERCIEMENTS}

Nous remercions le Dr Clarisse Barro, sélectionneur à de l'INERA, station de Saria, pour nous avoir fourni le sorgho (Sorghum bicolor). Nos remerciements vont également à l'endroit de Blandine Nacoulma et Oumarou Sambaré, Botanistes du Laboratoire de Biologie Végétale, Université Joseph KiZerbo pour l'identification des plantes.

\section{REFERENCES}

Adeniyi SA, Orjiekwe CL, Josiah SJ. 2009. Kinetics of the reaction of compound III of horseradish peroxidase with hydrogen peroxide and NADPH. Int. J. Biol. Chem. Sci., 3(3): 415-421. DOI: http://dx.doi.org/10.4314/ijbcs.v3i3.4535 0

Agostini E, Hernandez-Ruiz J, Arnao MB, Milrad SR, Tigier HA, Acosta M. 2002. A peroxidase isoenzyme secreted by Turnip (Brassica napus) hairy - root Cultures: inactivation by hydrogen peroxide and application in diagnostic Kits. Biotechnol. Appl. Biochem., 35(1): 1-7.

$$
\text { DOI: }
$$
https://doi.org/10.1042/BA20010049

Altın S, Tohma H, Gülçin İ, Köksal E. 2017. Purification, characterization and inhibition sensitivity of peroxidase from wheat (Triticum aestivum ssp. vulgare). International Journal of Food Properties, 20(9): 1949-1959. DOI: https://doi.org/10.1080/10942912.2016.1 225308

Altay A, Koktepe T, Durmaz L, Topal F, Gülçin İ, Köksal E. 2018. Purification and selected biochemical properties of 
peroxidase from cress (Lepidium sativum sub sp. sativum). International Journal of Food Properties, 21(1): 2610-2621. DOI:

https://doi.org/10.1080/10942912.2018.1 540989

Al-Senaidy AM, Ismael MA. 2011. Purification and characterization of membrane-bound peroxidase from date palm leaves (Phoenix dactylifera L.). Saudi J Biol Sci., 18(3): 293-298. DOI: https://doi.org/10.1016/j.sjbs.2011.04.00 5

Athon GE, Barett DM. 2002. Kinetic parameters for the thermal inactivation of quality-related enzymes in carrots and potatoes. J. Agric. Food Chem., 50(14): 4119-4125.

DOI: https://doi.org/10.1021/jf011698i

Benkeblia N, Shiomi N. 2004. Chilling effect on soluble sugars, respiration rate, Total phenolics, peroxidase activity and dormancy of onion bulbs. Sci. Agric., (Piraciaba, Braz), 61(3): 281 - 285. DOI: http://dx.doi.org/10.1590/S010390162004000300007

Diao M, Ouédraogo N, Baba-Moussa L, Savadogo PW, N'Guessan AG, Bassolé IHN, Dicko MH. 2011. Biodepollution of wastewater containing phenolic compounds from leather industry by plant peroxidases. Biodegradation, 22(2): 389-396. DOI: https://doi.org/10.1007/s10532-0109410-8

Diao M, Dibala CI, Ayékoué BN, Dicko MH. 2018. Biochemical characterization of Burkina red radish (Raphanus sativus) peroxidase. J. Appl. Biosci., 125: 1251812530.

DOI: http://dx.doi.org/10.4314/jab.v125i1.2

Dicko MH, Hilhorst R, Gruppen H, Traoré AS, Laana C, Van Berkel WJH, Voragen AGJ. 2002. Comparison of content in phenolic compounds, polyphenol oxidase and peroxidase in grains of fifty sorghum varieties from Burkina Faso. $J$. Agric. Food Chem., 50(13): 3780-3788. DOI: https://doi.org/10.1021/jf011642o

Diop CT, Messori L, Heimler D, Nali C, Lorenzini G. 2019. Time course of antioxidant enzymes in the paraquat- resistant and tolerant Heary fleabane

(Conyza bonariensis) biotypes in response to ozone exposure and paraquat application. Int. J. Biol. Chem. Sci., 13(2): $\quad 802-810 . \quad$ DOI: https://dx.doi.org/10.4314/ijbcs.v13i2.18

Kim SS, Lee DJ. 2005. Purification and characterization of a cationic peroxidase $\mathrm{C}_{S}$ in Raphanus sativus. Journal of Plant Physiology, 162(6): 609-617. DOI: https://doi.org/10.1016/j.jplph.2004.10.0 04

Leon JC, Alpeeva IS, Chubar TA, Galaev IY, Csoregi E, Sakharov I, Sakharov IY. 2002. Purification and substrate specificity of peroxidase from sweet potato tubers. Plant Sci., 163(5): 10111019.

DOI: https://doi.org/10.1016/S01689452(02)00275-3

Lim YY, Ong L, Loh TP, Sethi SK, Sng AAJ, Loke KY, Halsall DJ, Hughes IA, Lee YS. 2018. A diagnostic curiosity of isolated androstenedione elevation due to autoantibodies against horseradish peroxidase label of the immunoassay. Clinica Chimica Acta, 476: 103-106. DOI: https://doi.org/10.1016/j.cca.2017.11.025

Lu Y, Ye W, Yang Q, Yu J, Wang Q, Zhou P, Wang C, Xue D, Zhao S. 2016. Threedimensional hierarchical porous $\mathrm{Pt} \mathrm{Cu}$ dendrites: A highly efficient peroxidase nanozyme for colorimetric detection of $\mathrm{H}_{2} \mathrm{O}_{2}$, Sensors and Actuators B. Chemical, 230: 721-730. DOI: https://doi.org/10.1016/j.snb.2016.02.13 0

Mbouobda HD, fotso, Djeuani C, Baliga MO, Omokolo DN. 2014. Comparative evaluation of enzyme activities and phenol content of Irish potato (Solanum tuberosum) grown under EM and IMO manures Bokashi. Int. J. Biol. Chem. Sci., 8(1): 157-166. DOI: http://dx.doi.org/10.4314/ijbcs.v8i1.15

Mika A, Lüthje S. 2003. Properties of guaiacol peroxidase activities isolated from corn root plasma membranes. Plant Physiol., 132: 1489-1498. DOI: https://doi.org/10.1104/pp.103.020396 
Neves VA. 2002. Ionically Bound Peroxidase from Peach Fruit. Braz. Arch. Biol. Technol., 45(1): 7-16. DOI: http://dx.doi.org/10.1590/S151689132002000100002

Nwobodo EI, Nwosu DC, Ogbodo SO, Ugwuene FO, Ihim AC, Onuabuchi Ani N, Nnodim JK, ANI O. 2018. Effects of Azadirachta indica leaf aqeous extract on the antioxidant enzymes in paracetamol-induced hepatotoxicity in Wistar rats. Int. J. Biol. Chem. Sci., 12(1): $1-10 . \quad$ DOI: https://dx.doi.org/10.4314/ijbcs.v12i1.1

Onsa GH, Saari NB, Selamat J, Bakar J. 2004. Purification and characterization of membrane-bound peroxidases from Metroxylon sagu. Food Chem., 85(3): 365-376.

DOI: https://doi.org/10.1016/j.foodchem.2003. 07.013

Pandey S, Fartyal D, Agarwal A, Shukla T, James D, Kaul T, Negi YK, Arora S, Reddy MK. 2017. Abiotic stress tolerance in plants: myriad roles of ascorbate peroxidase. Frontiers in Plant Science, 8: 581-593. DOI: https://doi.org/10.3389/fpls.2017.00-581

Pinto MST, Ribeiro JM, Pinheiro de Araújo F, Franklin de Melo N, Fernandes KVS. 2015. Purification and characterization of a peroxidase present in xilopodium exsudates of umbu plants (Spondias tuberosa A.). Afr. J. Biotechnol., 14(21): 1838-1845.

DOI: https://doi.org/10.5897/AJB2015.14521

Quiroga M, Guerro C, Botella MA, Barcelo A, Amay I, Medina MI, Alonso FJ, de Frochetti SM, Tigier H, Valpuesta V. 2000. A tomato peroxidase involved in the synthesis of lignin and suberin. Plant Physiol., 122: 1119-1127. DOI: https://doi.org/10.1104/pp.122.4.1119

Santos de Araujo B, de Oliveira JO, Machado SS, Pletsch M. 2004. Comparative studies of the peroxidases from hairy roots of Daucus carota, Ipomoea batatas and Solanum aviculare. Plant Sci., 167(5): 1151-1157. DOI: https://doi.org/10.1016/j.plantsci.2004.06 .015

Sonet J, Bierla K, Bulteau AL, Lobinski R, Chavatte L. 2018. Comparison of analytical methods using enzymatic activity, immunoaffinity and seleniumspecific mass spectrometric detection for the quantitation of glutathione peroxidase 1. Analytica Chimica Acta, 1011: 11-19. DOI: https://doi.org/10.1016/j.aca.2018.01.068

Tao YM, Wang S, Luo HL, Yan WW. 2018. Peroxidase from jackfruit: Purification, characterization and thermal inactivation. Int. J. Biol. Macromol., 114: 898-905. DOI: https://doi.org/10.1016/j.ijbiomac.2018.0 4.007

Wang L, Burhenne K, Kristensen BK, Rasmussen SK. 2004. Purification and cloning of a Chinese red radish peroxidase that metabolize pelargonidin and forms a gene family in Brassicaceae. Gene, $\quad \mathbf{3 4 3}(2)$ : $\quad 323-335 . \quad$ DOI: https://doi.org/10.1016/j.gene.2004.09.0 18

Wang S, Wang Q, Fan X, Xu J, Zhang Y, Yuan J, Jin H, Cavaco-Paulo A. 2016. Synthesis and characterization of starchpoly (methyl acrylate) graft copolymers using horseradish peroxidase. Carbohydrate Polymers, 136: 1010$1016 . \quad$ DOI: https://doi.org/10.1016/j.carbpol.2015.09 .110

$\mathrm{Xu}$ TS. 2016. Bioconjugation of peroxidaselike nanostructures with natural enzyme for in-situ amplified conductometric immunoassay of tissue polypeptide antigen in biological fluids. Biochemical Engineering Journal, 105: 36-43. DOI: https://doi.org/10.1016/j.bej.2015.08.018

Zia MA, Kousar M, Ahmed I, Iqbal HMN, Abbas RZ. 2011. Comparative study of peroxidase purification from apple and orange seeds. Afr. J. Biotechnol., 10(33): 6300-6303.

DOI: https://doi.org/10.5897/AJB10.2675. 